PROCEEDINGS OF THE

AMERICAN MATHEMATICAL SOCIETY

Volume 137, Number 9, September 2009, Pages 2943-2951

S 0002-9939(09)09909-2

Article electronically published on April 8, 2009

\title{
CORE PARTITIONS AND BLOCK COVERINGS
}

\author{
JØRN B. OLSSON
}

(Communicated by Ken Ono)

\begin{abstract}
We prove some new results on core partitions and apply them to describe explicitly all block coverings in symmetric groups.
\end{abstract}

\section{INTRODUCTION}

A number of new results about core partitions have been proved recently ( 2 , 3, [9], 12]). For $s \in \mathbb{N}$ an $s$-core is by definition an integer partition without hooks of length $s$. This type of partition first occurred in the modular representation theory of symmetric groups, where $s$-cores label $s$-blocks of defect 0 in the case where $s$ is a prime. In the study of relations between blocks for different primes in symmetric groups it is of interest to study partitions which are simultaneously $s$ - and $t$-cores for different $s, t$ ([13]).

In this paper we present results of a new type on core partitions. The motivation for these results lies is the desire to classify completely the possible block coverings in symmetric groups, but the results may also have applications of a different nature.

There is a general question about possible equalities between (unions of) blocks in a finite group for different primes. It is related to the Navarro-Willems question about the possible equality of (the set of irreducible characters in) two blocks $B_{s}$ and $B_{t}$ for different primes ([10]). Precisely formulated the question is: When is, for a fixed pair of different primes $s, t$ dividing $|G|$ ( $G$ a finite group) the set of irreducible characters in a $t$-block $B_{t}$ of $G$ of positive defect equal to a union of the sets of irreducible characters in some $s$-blocks? We refer to this as a block covering of $B_{t}$. We call a block covering trivial if the $s$-blocks occurring in the union all have defect 0 .

If there is only one $s$-block in the covering, we have the special case of a block equality. In [10] it was conjectured that all block equalities are trivial. This is false in general, as noted first by C. Bessenrodt, but it is true in the case of symmetric groups ([13], Corollary 2.8). This shows that block equalities in the symmetric groups are parametrized by $(s, t)$-cores as defined below. There are only finitely many $(s, t)$-cores, given $s$ and $t([1])$. This is a special case $(w=0)$ of one of our main results, Theorem 4.2 below.

There are examples of non-trivial block coverings in finite groups. Such examples occur in some sporadic simple groups: $M_{11}, M_{22}, M_{23}, M_{24}, C_{2}, J_{4}, B, M$. (See e.g. Section 3 in [5.) Also there are examples in some quasisimple groups of Lie

Received by the editors January 6, 2009

2000 Mathematics Subject Classification. Primary 20C30, 05A17.

(C)2009 American Mathematical Society

Reverts to public domain 28 years from publication 
type, where $t$ is the defining characteristic. In [5] all occurrences of block coverings of the principal $t$-block in arbitrary finite groups are listed.

In this note we want to show that (apart from some trivial exceptions for $n \leq 4$ ) a block covering in a symmetric groups $S_{n}$ is only possible when the $s$-blocks are all of weight (defect) 0, i.e. when these block coverings are all trivial (Theorem 3.7). It is also possible to describe very explicitly the occurrences of the trivial coverings (Theorem 4.2). These results are all obtained in the more general case, where $s$ and $t$ are not necessarily prime numbers, but only relatively prime positive integers, both not equal to 1 .

In the symmetric group $S_{n}$ the irreducible characters are labelled canonically by the partitions of $n$, and the distribution of the irreducible characters into $s$-blocks is given by a combinatorial condition on their labels, still referred to as the Nakayama conjecture. See [6], 6.2.21. This motivates us to work with blocks of partitions, i.e. the set of labels of all the irreducible characters in a block. As in earlier papers ([13, 4]) the primeness of $s$ and $t$ is not essential, and considering the results of [8] this may still have some character-theoretic relevance.

For the basic facts concerning partitions, hooks and blocks of partitions, we refer to [6], Chapter 2 or [11, Chapter 1 . One may get to the $s$-core $\lambda_{(s)}$ of a partition $\lambda$ by removing a series of $s$-hooks (hooks of length $s$ ) until all $s$-hooks are removed. The $s$-core is independent of the order in which the $s$-hooks are removed. A partition $\lambda$ has by definition $s$-weight $w$ if you need to remove exactly $w s$-hooks to get to its $s$-core. It also equals the number of hooks in $\lambda$ of length divisible by $s\left([\underline{6}, 2.7 .40)\right.$. We denote this number by $w_{s}(\lambda)$.

A partition has $s$-weight 0 if and only if it has no hook of length $s$. Such a partition is called an $s$-core. Two partitions of $n$ are said to be in the same $s$-block $B_{s}$ if they have the same $s$-core. In this case, the weight $w\left(B_{s}\right)$ of the block is the common $s$-weight $w_{s}(\lambda)$ of the partitions $\lambda$ in $B_{s}$ and the core of $B_{s}$ is the common $s$-core of all the partitions in $B_{s}$. In particular, an $s$-core partition forms an $s$-block of weight 0 by itself.

The hook structure of a partition is conveniently determined by the set of its first column hook lengths, or more generally any of its $\beta$-sets; see [11, Chapter 1. Generally, a $\beta$-set is a finite subset $X$ of $\mathbb{N}_{0}=\{0,1,2, \cdots\}$.

For $i \geq 0$ let $X^{+i}$, the $i$ 'th shift of $X$, be the $\beta$-set which is obtained from $X$ in the following way: It is the union of the set $\{0,1, \cdots, i-1\}$ and the set obtained from $X$ by adding $i$ to all its elements. In particular $X^{+0}=X$. The $\beta$-set $\{0,2,3,6,7\}$ equals $\{1,2,5,6\}^{+1}$.

Let $\lambda$ be a partition. Let $\beta(\lambda)$ be the $\beta$-set consisting of all first column hook lengths of $\lambda$. Thus if $\lambda=(3,3,1,1)$, then $\beta(\lambda)=\{1,2,5,6\}$. A $\beta$-set on the form $\beta(\lambda)^{+i}$ is called a $\beta$-set for $\lambda$.

Let $s \in \mathbb{N}$. The $s$-abacus is defined as follows: It has $s$ runners numbered $0,1, \cdots, s-1$ running from north to south. On the $i$-th runner we place all nonnegative integers of residue $i$ modulo $s$ in increasing order. A $\beta$-set $X$ (for a partition $\lambda$ ) may be represented by a bead configuration on the $s$-abacus by placing beads in the positions given by the numbers in the $\beta$-set. We refer to this also as the $s$-abacus for $X$.

For example, $X=\{1,3,7,10,11,12\}$, a $\beta$-set for the partition $(7,7,7,5,2,1)$ of 29 , is represented by the following bead configuration on the 3 -abacus: 


$\begin{array}{ccc}0 & \mathbf{1} & 2 \\ \mathbf{3} & 4 & 5 \\ 6 & \mathbf{7} & 8 \\ 9 & \mathbf{1 0} & \mathbf{1 1} \\ \mathbf{1 2} & 13 & 14\end{array}$

The $s$-core of a partition may also be determined from a $\beta$-set on the $s$-abacus. This is due to the following well-known fact:

Lemma 1.1. Suppose that $X$ is a $\beta$-set for the partition $\lambda$. Then $\lambda$ contains a hook of length $s$ if and only if there exists an $h \in X$ such that $h-s \geq 0$ and $h-s \notin X$. In this case $X \cup\{h-s\} \backslash\{h\}$ is a $\beta$-set for a partition obtained by removing an $s$-hook from $\lambda$.

Thus removing/adding an $s$-hook of $\lambda$ corresponds to moving a bead one position up/down to an empty position on one of the runners. You get a $\beta$-set for the $s$-core $\lambda_{(s)}$ of $\lambda$ when all beads are in the highest possible position.

Corollary 1.2. Suppose that $X$ is a $\beta$-set for the partition $\lambda$. Then $\lambda$ is an s-core if and only if for all $h \in X$ we have: If $h-s \geq 0$, then $h-s \in X$.

We study then generally the situation where a $t$-block $B_{t}$ of positive weight $w\left(B_{t}\right)=w>0$ in $S_{n}$ is a union of $k \geq 1$ different $s$-blocks, where we assume that $s$ and $t$ are relatively prime:

$$
B_{t}=\bigcup_{i=1}^{k} B_{s}^{(i)} .
$$

This is called a block covering (of a $t$-block by $s$-blocks) in $S_{n}$. We call $w\left(B_{t}\right)$ the weight of the covering.

Examples of coverings. Let $t=3$ and $s=11$. Consider the 3-core $\kappa_{1}=$ $\left(7,5,3,2^{2}, 1^{2}\right)$ of 21 . The 3 -block of weight 1 with this core consists of the partitions $\left(10,5,3,2^{2}, 1^{2}\right),\left(7,5,4^{2}, 2,1^{2}\right)$ and $\left(7,5,3,2^{2}, 1^{5}\right)$ of 24 , which are all 11-cores. As we shall see later, a 3 -block of weight 2 with 3 -core $\kappa_{2}=\left(4,2,1^{2}\right)$ also consists of partitions of 14, all of which are 11-cores. Thus we have two examples of a 3block being a union of 11-blocks. We shall also see that the decisive facts for these coverings to occur is that $\kappa_{1}$ is an 8 -core and $\kappa_{2}$ is a 5 -core!

In the next section we present our new results on core partitions. Then it will be shown in section 3 that for $n \geq 5$ and $s, t \neq 1$ relatively prime all block coverings in $S_{n}$ are trivial. In the final section we describe explicitly all the possible trivial block coverings.

\section{Some GenERAL RESUlTS ON CORES}

We assume that $s, t$ are positive integers, not necessarily relatively prime. An $(s, t)$-core is a partition which is both an $s$-core and a $t$-core.

It is known that if $t \mid s$, then a $t$-core is also an $s$-core. In fact more is true. Again let $w_{t}(\lambda)$ denote the $t$-weight of the partition $\lambda$, for a given $t \in \mathbb{N}$. We have

Lemma 2.1. Let $v, t \in \mathbb{N}$ and let $\lambda$ be a partition. Then

$$
v \cdot w_{v t}(\lambda) \leq w_{t}(\lambda)
$$

In particular $w_{t}(\lambda)=0 \Rightarrow w_{v t}(\lambda)=0$. 
Proof. Using the $t$-abacus it is easy to see that the removal of a $v t$-hook may be obtained by a successive removal of $v t$-hooks. From this the lemma follows.

Theorem 2.2. An $(s, t)$-core is also an $(s+t)$-core and thus an $(s+w t)$-core for all $w \geq 0$.

Proof. Let $\rho$ be an $(s, t)$-core and let $X$ be its set of first column hook lengths. Suppose that $h \in X$ and that $h-(s+t) \geq 0$. We show that $h-(s+t)=(h-s)-t \in X$. Then Corollary 1.2 implies that $\rho$ is an $(s+t)$-core.

Since $\rho$ is an $s$-core and $(h-s) \geq 0$, we get $h_{1}=h-s \in X$. Since $\rho$ is a $t$-core and $h_{1}-t \geq 0$, we get $h_{1}-t=(h-s)-t \in X$, as desired.

Corollary 2.3. Suppose that $w \geq 0$ satisfies $0<w t<s$ and that $s_{1}=s-t w$. Then an $\left(s_{1}, t\right)$-core is also an $(s, t)$-core.

Theorem 2.4. Suppose that $\rho_{w}$ is obtained from the $(s, t)$-core $\rho$ by adding $w$ t-hooks. Then $\rho_{w}$ is an $(s+t w)$-core.

Remark. Obviously if $w>0$, then $\rho_{w}$ is not a $t$-core. It also need not be an $s$-core.

Proof. Consider first the case where $t \mid s$, say $s=v t$. By Lemma 2.1, then, an $(s, t)$ core is the same as a $t$-core. We now have that $w_{t}\left(\rho_{w}\right)=w$. Moreover $s+w t=$ $(v+w) t$. If $\rho_{w}$ is not an $(s+w t)$-core, then $w_{s+w t}\left(\rho_{w}\right)=w_{(v+w) t}\left(\rho_{w}\right)>0$, and by Lemma 2.1 we then have $v+w \leq(v+w) w_{(v+w) t}\left(\rho_{w}\right) \leq w_{t}\left(\rho_{w}\right)=w$. This implies that $v=0$, a contradiction.

Thus we may assume that $t \nmid s$. Let $Y$ be a $\beta$-set for the partition $\rho_{w}$ of $n+w t$ and let $X$ be a $\beta$-set for the $(s, t)$-core $\rho$ of $n$ having the same cardinality as $Y$. For $0 \leq i \leq t-1$, let

$$
X_{i}=\left\{h \in X \mid h \equiv_{t} i\right\}, Y_{i}=\left\{k \in Y \mid k \equiv_{t} i\right\} .
$$

Each $Y_{i}$ is a $\beta$-set for a partition (of $w_{i}$, say) in the $t$-quotient of $\rho_{w}$. Then $w=w_{0}+\ldots+w_{t-1}$.

Representing $Y_{i}$ and $X_{i}$ on the $i$-th runner of the $t$-abacus, we have that $Y_{i}$ is obtained from $X_{i}$ by a sequence of $w_{i}$ moves, where each move consists of moving a bead to an empty position immediately below it. This shows that we have the following facts:

(I) If $h^{\prime} \in X_{i} \backslash\left(X_{i} \cap Y_{i}\right)$, then $w_{i} \geq\left|\left\{h \in X_{i} \mid h \geq h^{\prime}\right\}\right|$.

(Indeed, since $h^{\prime} \notin Y_{i}$ the bead representing it has to be moved. To do this we first have to move the beads below it on the runner.)

(II) If $k \in Y_{i}$ and $w^{\prime}=\min \left\{v \geq 0 \mid k=h+v t\right.$ for some $\left.h \in X_{i}\right\}$, then $w_{i} \geq w^{\prime}$.

(Indeed we need at least $w^{\prime}$ moves to move a bead in $X_{i}$ to the position occupied by $k$.)

Suppose now that $a:=k-(s+w t) \geq 0$ for some $k \in Y_{i}$. We want to show $a \in Y$. Put $a_{v}=a+v t$, for $v=0, \ldots, w$ so that $a_{0}=a, a_{w}=k-s$. Put $b_{v}=a_{v}+s$, so that $b_{w}=k$. The $a_{v}$ are all on the same runner, say runner $j$, and the $b_{v}$ are on runner $i$. We have $i \neq j$, since $t \nmid s$.

Choose a minimal $w^{\prime} \geq 0$ for which there exists an $h \in X$ such that $k=h+w^{\prime} t$. By (II) above we know $w_{i} \geq w^{\prime}$. Now $h=k-w^{\prime} t=b_{w-w^{\prime}}$. Thus $h=b_{w-w^{\prime}} \in X$. Since $X$ is a $\beta$-set for a $t$-core we get $b_{v} \in X$ for $0 \leq v \leq w-w^{\prime}$. This shows that $a_{v} \in X$ for $0 \leq v \leq w-w^{\prime}$, since $X$ is also a $\beta$-set for an $s$-core.

We assume $a \notin Y_{j}$ and seek a contradiction. Applying (I) above to $a \in X_{j}$ we then get $w_{j} \geq w-w^{\prime}$. Thus $w \geq w_{i}+w_{j} \geq w^{\prime}+\left(w-w^{\prime}\right)=w$, and therefore 
we have the equalities $w_{i}=w^{\prime}, w_{j}=w-w^{\prime}$. The last equality shows that $Y_{j}=$ $X_{j} \cup\left\{a+\left(w-w^{\prime}\right) t\right\} \backslash\{a\}$.

This is not possible since $a+\left(w-w^{\prime}\right) t=h-s \in X$.

As a kind of converse to the theorem we have:

Theorem 2.5. Let $w \geq 0$ be given and assume that $\rho$ is a partition with the following property:

$(*)$ Whenever $\rho_{w}$ is obtained from $\rho$ by adding $w$ t-hooks, $\rho_{w}$ is an $(s+t w)$-core.

Then $\rho$ is an $s$-core.

Proof. Let $X$ be a sufficiently large $\beta$-set for $\rho$ and let $X_{i}$ as before $(0 \leq i \leq t-1)$ be the subset of $X$ represented on the $i$ 'th runner. Let $h \in X$ satisfy that $h-s \geq 0$. We want to show that $h-s \in X$. Let $h^{\prime}=h+w t$.

If $h^{\prime} \notin X$, then $Y=X \cup\left\{h^{\prime}\right\} \backslash\{h\}$ is a $\beta$-set for a partition $\rho_{w}$ which is obtained from $\rho$ by adding $w t$-hooks. By assumption $\rho_{w}$ is an $(s+w t)$-core. Since $h^{\prime}-w t-s=h-s \geq 0$, we get $h-s \in Y$ and thus $h-s \in X$.

If $h^{\prime} \in X$, say $h^{\prime} \in X_{i}$, then let us choose some $j \neq i$ and a $k \in X_{j}$ such that $k+w t \notin X_{j}$. Put $Y=X \cup\{k+w t\} \backslash\{k\}$. We then have that $h^{\prime}-w t-s=h-s \in Y$, since $Y$ is a $\beta$-set for an $(s+w t)$-core. If $h-s \notin X$, then $h-s=k+w t$. In that case $k \notin Y$. But $h \in Y$ and $k=h-(w t+s) \geq 0$. Since $Y$ is a $\beta$-set for an $(s+w t)$-core, this is impossible.

\section{BLOCK COVERINGS IN $S_{n}$}

We assume throughout this section that $s$ and $t$ are relatively prime and both not equal to 1 . In the previous section there was no assumption on $s$ and $t$ being relatively prime, and certainly it will be possible to prove results on block coverings in $S_{n}$ in the general case. However these are going to be of an entirely different nature than the ones obtained below, which are not correct in general. One essential difference is that in the case where $s$ and $t$ are relatively prime there are only finitely many $(s, t)$-cores. If $s$ and $t$ have a common divisor $v \neq 1$, then any $v$-core is an $(s, t)$-core and there exist infinitely many $v$-cores and thus infinitely many $(s, t)$ cores. Also considering the original question for arbitrary groups, the assumption about relative primeness appears to be reasonable.

We define (for $s, t$ relatively prime)

$$
a_{s, t}=\frac{\left(s^{2}-1\right)\left(t^{2}-1\right)}{24} .
$$

Then it is known that $a_{s, t}$ is the maximal number $n$ such that there exists a partition of $n$ which is an $(s, t)$-core. (See e.g. [13, Theorem 4.1.) There is a unique $(s, t)$-core, denoted $\kappa_{s, t}$ of $a_{s, t}$. In [14] it is shown that any $(s, t)$-core is contained in $\kappa_{s, t}$. The partition $\kappa_{s, t}$ is also the unique minimal $(s, t)$-good partition. This is by definition an $s$-core such that the partitions in an $s$-block of weight 1 all have the same $t$-core. These partitions are described in detail in [13].

Assume that we have a block covering in $S_{n}$,

$$
B_{t}=\bigcup_{i=1}^{k} B_{s}^{(i)} \text {. }
$$


If $k=1$, we have a block equality and this is then trivial, [13, Corollary 2.8. We may then assume $k \geq 2$, which implies $w=w\left(B_{t}\right)>0$. Also we let $\rho$ denote the core of the block $B_{t}$, so it is a $t$-core. We keep this notation in the following.

The main result in this section is

Theorem 3.1. Suppose that $s, t$ are relatively prime, both $\neq 1$. There exists a non-trivial covering of a $t$-block by s-blocks in $S_{n}$ if and only if $n, s, t$ satisfy one of the following conditions:

- $(n, s, t)=(3,2,3)$.

- $(n, s, t)=(4,3,2)$.

We give a series of lemmas, leading up to a proof of the theorem.

Lemma 3.2. If $S_{n}$ has only one $t$-block, $t>1$, then we have one of the following cases:

- $t=2, n=2$ or $n=4$.

- $t=3, n=3$.

In these cases we have a covering of the (unique) t-block by s-blocks for any $s$.

Proof. Let $n>1$. For $n<5$ there are only the cases listed above. For $n \geq 5$, it can easily be shown that at least two of the partitions $(n),\left(1^{n}\right),(n-2,2),\left(n-2,1^{2}\right),(n-$ $1,1)$ have different $t$-cores. The final statement is trivial from the definition.

Since for $n>1, S_{n}$ has an $s$-block of positive weight if and only if $s \leq n$, the lemma shows that the if part of Theorem 3.1 is true. To prove the only-if part we show that for $n \geq 5$, all block coverings in $S_{n}$ of a $t$-block by $s$-blocks are trivial. This is done in Lemma 3.6 below.

Thus from now on we assume $n \geq 5, s, t>1$.

We know that each $B_{s}^{(i)}$ has weight 0 or 1 by [13, Theorem 2.5. From [13, Theorem 5.3, we get

Lemma 3.3. If the block covering is non-trivial, i.e. if some $B_{s}^{(i)}$ is of weight 1 , then $\rho$ is an explicitly given $(s, t)$-core and $|\rho|=a_{s, t}-s t+s+t$.

Lemma 3.4. The partition $\rho$ is also an s-core. In particular $|\rho| \leq a_{s, t}$.

Proof. Consider e.g. $B_{s}=B_{s}^{(1)}$. If $B_{s}$ has weight 0 , then $\lambda \in B_{s}$ is an $s$-core. Moreover $\lambda_{(t)}=\rho$, as $\lambda \in B_{t}$. By Theorem 1 of [12] $\rho$ is again an $s$-core, so that $\rho$ is in fact an $(s, t)$-core. If $B_{s}$ has weight 1 , then by Lemma $3.3 \rho$ is an $s$-core. The last statement follows e.g. from [13, Theorem 4.1.

Lemma 3.5. If some $B_{s}^{(i)}$ has weight 1 , then $w \geq(s-1)$.

Proof. Assume that $B_{s}=B_{s}^{(1)}$ has weight 1. The $(s-)$ core of $\kappa$ of the partitions in $B_{s}$ is then an $(s, t)$-good partition. By [13, Theorem 5.1, we have $|\kappa|=a_{s, t}+t^{2} v$ for some $v \geq 0$. Thus $n=a_{s, t}+t^{2} v+s$. We get by Lemma 3.3,

$$
w=\frac{n-|\rho|}{t}=\frac{t^{2} v+s+s t-s-t}{t}=(s-1)+t v,
$$

finishing the proof.

Lemma 3.6. All $B_{s}^{(i)}$ 's have weight 0. 
Proof. Assume that some $B_{s}^{(i)}$ has weight 1 , say $B_{s}=B_{s}^{(1)}$. Consider the partition $\lambda$ obtained from $\rho$ by adding $w t$ nodes in the first row. Then $\lambda \in B_{t}$ and thus also in some $B_{s}^{(j)}, 1 \leq j \leq k$. Obviously it is possible to remove at least $v=\left\lfloor\frac{w t}{s}\right\rfloor$ $s$-hooks from the first row of $\lambda$. Thus

$$
w_{s}(\lambda) \geq v \geq\left\lfloor\frac{(s-1) t}{s}\right\rfloor
$$

by Lemma 3.5. On the other hand $w_{s}(\lambda)=w\left(B_{s}^{(j)}\right) \leq 1$. Thus $\left\lfloor\frac{(s-1) t}{s}\right\rfloor \in\{0,1\}$. The case $\left\lfloor\frac{(s-1) t}{s}\right\rfloor=0$ is not possible, as $s, t \neq 1$. We thus get $s<(s-1) t<2 s$. This is satisfied for $t=2$.

If $t \geq 3$, we have $3(s-1)<2 s$, forcing $s=2$ and then $t=3$. This case $s=2, t=3$ is easily handled. Indeed, $\rho=(0)$ by Lemma 3.3. Since we assume $n \geq 5$ we get $w \geq 2$. The partition $(3 w)$ is in some $B_{s}^{(j)}$ and has 2-core (0) or (1), depending on the parity of $w$. This forces its 2-weight to be at least 2, a contradiction.

Consider the case $t=2$. Then by [13], Theorem 5.3, $\rho=\left(s^{\prime}-1, s^{\prime}-2, \ldots, 1\right)$, where $s^{\prime}=(s-1) / 2$. By Lemma $3.5 w \geq s-1 \geq 2$. Add $2 w-(s-1)$ to the largest part of $\rho$ and $(s-1)$ to the second largest part of $\rho$. This partition is in $B_{t}$ since $(s-1)$ is even. For $s^{\prime} \geq 3$ the partition has at least $s$-weight 2 . Indeed it is then possible to remove an $s$-hook from the second row and then remove an $s$-hook from the first row. This is a contradiction. We need to consider $s^{\prime}=1,2$, i.e. $s=3,5$.

If $s=3$, then $\rho=(0)$ and $n=2 w$. Since $n \geq 5$ we get $w \geq 3$. But then the partition $\left(w^{2}\right) \in B_{t}$ has $s$-weight at least 2 , a contradiction.

If $s=5$, then $\rho=(1)$ and $w \geq 4$. If $w \geq 5$, then $(w+1, w) \in B_{t}$ has $s$-weight at least 2. If $w=4$, then $n=9$. The core of $B_{s}$ is $(5,2)$-good and thus must be a partition of 3 or 7 by [13, Theorem 3.1. This is not possible.

Lemma 3.6 depended on the assumption that $n \geq 5, t>1$. It shows the only-if part of Theorem 3.1 and thus concludes its proof.

Let us formulate explicitly the following:

Theorem 3.7. Suppose that $n \geq 5$ and that $s, t \neq 1$ are relatively prime. Then any block covering

$$
B_{t}=\bigcup_{i=1}^{k} B_{s}^{(i)}
$$

of a t-block by s-blocks in $S_{n}$ is trivial.

The principal block in a finite group is the block containing the trivial character. In our setup it is thus the block containing the partition $(n)$.

Corollary 3.8. In a block covering as in Theorem $3.7, B_{t}$ is not the principal block of $S_{n}$ unless $s>n$.

Proof. If $B_{t}$ is the principal block and the covering is trivial, then $B_{s}^{(i)}=\{(n)\}$ for some $i$. Thus the principal $s$-block of $n$ has weight 0 , forcing $s>n$.

\section{Describing All Possible Block COVERINGS}

We are still assuming that $s$ and $t$ are relatively prime $\neq 1$ and are going to give a parametrization of all possible block coverings in symmetric groups, given $s$ and $t$. The occurrences of non-trivial coverings (for $n=3,4$ ), as described explicitly in Theorem 3.1. We need then only to classify the trivial block coverings. 
Lemma 4.1. Suppose that $s, t$ are relatively prime. If we have a trivial block covering

$$
B_{t}=\bigcup_{i=1}^{k} B_{s}^{(i)}
$$

of a t-block of weight $w$ by $s$-blocks, then $w t<s$.

Proof. Assume that $s \leq w t$. Then $w>0$. Let $\lambda$ be obtained from the core $\rho$ of $B_{t}$ by adding $w t$ to its largest part. Then $\lambda$ is not an $s$-core, since $s \leq w t$. This contradicts our assumption.

Theorem 4.2. Suppose that $s, t$ are relatively prime. Let $B_{t}$ be a $t$-block of weight $w \geq 0$ with core $\rho$. Then $B_{t}$ is covered trivially by s-blocks if and only if $w t<s$ and $\rho$ is an $(s-w t)$-core.

Proof. Suppose first that $B_{t}$ is covered trivially by $s$-blocks. Then by Lemma 4.1 we get that $w t<s$. By assumption we know that all partitions in $B_{t}$ are $s$-cores. Then Theorem 2.5 shows that $\rho$ is an $(s-w t)$-core.

Conversely, if $w t<s$ and $\rho$ is an $(s-w t)$-core, then Theorem 2.2 shows that all partitions in $B_{t}$ are $s$-cores. This shows that $B_{t}$ is covered by $s$-blocks.

Theorem 4.3. Suppose that $n \geq 5$, that $s, t \neq 1$ and that $s, t$ are relatively prime. Suppose that $w t<s$ and put $s_{1}=s-w t$. The number of occurrences of a $t$-block of weight $w$ being covered by s-blocks equals the number of $\left(s_{1}, t\right)$-cores. This number is

$$
\left(\begin{array}{c}
s_{1}+t \\
t
\end{array}\right) /\left(s_{1}+t\right) .
$$

The maximal $n$ for which such a covering occurs is

$$
n=w t+\frac{\left(s_{1}^{2}-1\right)\left(t^{2}-1\right)}{24} .
$$

Proof. This is a consequence of Theorem 4.2 above, Theorem 3 in 1 and Theorem 4.1 in 13 .

Let us finally observe the following. If we have a block covering

$$
B_{t}=\bigcup_{i=1}^{k} B_{s}^{(i)}
$$

of a $t$-block of weight $w$ by $s$-blocks and $\rho$ is the core of $B_{t}$, then by Lemma $3.2 \rho$ is an $(s, t)$-core and by Theorem $4.2 \rho$ is an $\left(s_{1}, t\right)$-core, where $s_{1}=s-w t$. Now generally an $\left(s_{1}, t\right)$-core is also an $(s, t)$-core by Corollary 2.3. As described in [1, 13] $\beta$-sets for $(s, t)$-cores are represented by beads in a northeast justified subdiagram of the $(s, t)$-diagram. Now the $\left(s_{1}, t\right)$-diagram lies as a $w$-shifted subdiagram of the $(s, t)$ diagram, as illustrated by the example below. Here $s=17, t=5, w=2, s_{1}=7$.

The boldface numbers in this $(17,5)$-diagram are the numbers in the $(7,5)$ diagram:

$$
\begin{array}{ccccccccccccc}
12 & 7 & \mathbf{2} & & & & & & & & & & \\
29 & 24 & 19 & 14 & \mathbf{9} & \mathbf{4} & & & & & & & \\
46 & 41 & 36 & 31 & 26 & 21 & \mathbf{1 6} & \mathbf{1 1} & \mathbf{6} & \mathbf{1} & & & \\
63 & 58 & 53 & 48 & 43 & 38 & 33 & 28 & \mathbf{2 3} & \mathbf{1 8} & \mathbf{1 3} & \mathbf{8} & \mathbf{3}
\end{array}
$$


(The diagram is transposed compared to the convention of [13].) This observation shows that a northeast justified subdiagram of the $(s, t)$-diagram represents an $\left(s_{1}, t\right)$-core if and only if the first $w i$ entries in row $i$ are not in the subdiagram for all relevant $i$. It would perhaps be reasonable to call such a subdiagram $w$-shifted so that the number of possible coverings of an s-block of weight $w$ by $t$-blocks equals the number of $w$-shifted subdiagrams of the $(s, t)$-diagram.

\section{ACKNOWLEDGEMENT}

The author thanks Christine Bessenrodt for some useful comments on this paper.

\section{REFERENCES}

1. J. Anderson, Partitions which are simultaneously $t_{1}$ - and $t_{2}$-core, Discrete Math. 248 (2002), pp. 237-243. MR1892698(2002m:05021)

2. D. Aukerman, B. Kane, and L. Sze, On simultaneous s-cores/t-cores, to appear in Discrete Math., http://dx.doi.org/10.1016/j.disc.2008.06.024

3. C. Berg, B. Jones, and M. Vazirani, A bijection on core partitions and a parabolic quotient of the affine symmetric group, http://arxiv.org/abs/0804.1380

4. C. Bessenrodt and J.B. Olsson, Spin block inclusions, J. Algebra 306 (2006), pp. 3-16. MR.2271569 (2007j:20009)

5. C. Bessenrodt and J.P. Zhang, Block separations and inclusions, Advances Math. 218 (2008), pp. 485-495. MR2407943 (2009c:20015)

6. G. James and A. Kerber, The representation theory of the symmetric group, Encyclopedia of Mathematics and its Applications, 16, Addison-Wesley, Reading, Mass., 1981. MR644144 (83k:20003)

7. B. Kane, Simultaneous s-cores and t-cores, Master's thesis, 2002, Carnegie-Mellon University, unpublished.

8. B. Külshammer, J.B. Olsson and G.R. Robinson, Generalized blocks for symmetric groups, Invent. Math. 151 (2003), pp. 513-552. MR1961337 (2004c:20019)

9. R. Nath, On the t-core of an s-core partition, Integers 8 (2008), A28. MR 2425626

10. G. Navarro and W. Willems, When is a p-block a q-block? Proc. Amer. Math. Soc. 125 (1997), pp. 1589-1591. MR1423327 (97i:20013)

11. J.B. Olsson, Combinatorics and representations of finite groups, Vorlesungen aus dem FB Mathematik der Univ. Essen, Heft 20, 1993. This book is freely available on the author's homepage, http://www.math.ku.dk/ olsson/manus/comb_rep_all.pdf. MR1264418(95b:20020)

12. J.B. Olsson, A theorem on the cores of partitions, to appear in J. Combin. Theory Ser. A, http://dx.doi.org/10.1016/j.jcta.2008.08.004

13. J.B. Olsson and D. Stanton, Block inclusions and cores of partitions, Aequationes Math. 74 (2007), pp. 90-110. MR2355859 (2008j:20031)

14. J.A. Vandehey, A general theory of $(s, t)$-core partitions, http://arxiv.org/abs/0809.2134

Department of Mathematical Sciences, University of Copenhagen, UniversitetsParken 5, DK-2100 Copenhagen, Denmark

E-mail address: olsson@math.ku.dk 